\title{
Nonlinear Systems and Exponential Eigenfunctions
}

\author{
Paulo J. S. G. Ferreira, Member, IEEE
}

\begin{abstract}
It has been shown recently that homogeneous timeinvariant systems produce exponential outputs in response to similar exponential inputs, but that the concepts of "impulse response" and "frequency response" are of little use for their analysis. It was also asked whether there exist more general classes of systems with exponential eigenfunctions. In this letter, we recall that the concepts of impulse and frequency response can be useless even for certain linear, time-invariant systems. We briefly discuss the role of time-invariance, commuting linear systems, and conditions under which they have common eigenfunctions. Then we exhibit a class of nonlinear, nonhomogeneous, time-varying systems that still have exponential eigenfunctions. This class contains homogeneous time-invariant systems, finite impulse response (FIR) filters and generalized feedforward filters as special cases, and it shows that the exponential eigenfunction property does not imply linearity, homogeneity, or timeinvariance.
\end{abstract}

Index Terms - Commutativity, exponentials, fading memory, homogeneous time-invariant systems, linear time-invariant systems, nonhomogeneous systems, nonlinear systems.

\section{INTRODUCTION}

$\mathbf{L}$ INEAR time-invariant (LTI) systems have a well-known property: exponential inputs lead to similar exponential outputs. It has been pointed out [1] that homogeneous timeinvariant (HTI) systems share the same property, but that the concepts of "impulse response" and "frequency response" are of little use for the analysis of such systems. It is also asked in [1] whether there exist nonhomogeneous systems with the exponential eigenfunction property.

It is the aim of this letter to further discuss these points. In the next section, we mention the apparently not well-known fact that the concepts of impulse and frequency response can be useless even for certain LTI systems, mentioning past works that address this and other related issues.

By definition, LTI systems commute with delays (delaying the input merely delays the output by the same amount). The system that delays a signal by a constant amount is a simple example of a linear system with exponential eigenfunctions, and, roughly speaking, commuting linear systems generally have common eigenvectors or invariant subspaces. We comment on this fact, and we give a sufficient condition for the existence of a common eigenvector for commuting linear operators.

Can a system have exponential eigenfunctions without necessarily commuting with the delay operator? This brings us

Manuscript received June 8, 1999. This work was supported in part by the Fundação para Ciência e Tecnologia (FCT). The associate editor coordinating the review of this manuscript and approving it for publication was Dr. R. L. de Queiroz.

The author is with the Instituto de Engenharia Electrónica e Telemática, Universidade de Aveiro, 3810 Aveiro, Portugal (e-mail: pjf@inesca.pt).

Publisher Item Identifier S 1070-9908(99)08276-0. back to the question made in [1] concerning the existence of nonhomogeneous time-invariant systems with exponential eigenfunctions. The question has an affirmative answer, and it turns out that time-invariance is also not necessary.

Since a system is an arbitrary map between functions, even artificial input/output rules such as the following can be considered: the response to an exponential is the same exponential, the response to any other signal is a fixed nonexponential signal (for example, a step). This system is nonlinear, nonhomogeneous, and time-varying, and has the exponential eigenfunction property (by construction). The example is clearly devoid of any real interest. Note that, contrary to the one given in [1], it is not realizable. However, it raises another question: Are there interesting, potentially useful classes of systems that meaningfully generalize LTI systems and still possess exponential eigenfunctions? We will show that the answer is affirmative. The class of systems that we construct has the required properties and it contains some commonly used filter structures as special cases.

\section{RESUlTS}

A system is a map between signals (an operator). A functional is a map from a set of signals into the complex numbers. The arguments of nonlinear functionals or systems are written inside square brackets, but the brackets are dropped if the mapping is linear (round brackets are used if necessary). The operator that translates or delays the signal by $a$ is denoted by $D_{a}$, or simply $D$, if the value of $a$ is unimportant. A functional $F$ is time- or translation-invariant if $F[D x]=F[x]$ for all $D$, and a (linear or nonlinear) system $H$ is time-invariant if $D H[x]=H[D x]$, for all $D$.

Borrowing an idea from [2] and [3], consider the LTI system that maps the input signal $x$ into the constant signal $y=E x$, where $E[\cdot]$ is the average functional

$$
E x:=\lim _{N \rightarrow \infty} \frac{1}{2 N+1} \sum_{k=-N}^{N} x(k) .
$$

The impulse response of this system is the zero signal, but this fact is of little use because the system cannot be represented as a convolution. In fact, the system maps a nonzero constant signal into itself.

The existence of LTI systems that cannot be represented by convolutions has been noted by several authors, including [4], which draws on material found in [2]. The very simple example $y=x-E x$ appears in the more recent work [3]. Another recent reference is [5].

It is well known that any pair of commuting matrices has a common eigenvector [6]. By definition, an LTI sys- 
tem commutes with the delay $D_{a}$, and the exponentials are eigenfunctions of $D_{a}$. It is natural to ask if time-invariant systems have exponential eigenfunctions because they commute with delays, which have exponentials eigenfunctions. In other words, is time-invariance necessary? If it is not, it would be interesting to state conditions that guarantee that a commuting family of linear systems has common eigenfunctions.

The finite-dimensional problem is simple yet interesting. Consider periodic discrete signals of period $N$, and let $C_{a}$ denote the cyclic shift by $a$ samples. Any linear system acting on a $N$ th-dimensional space can be represented by a matrix, and if the system commutes with $C_{a}$ for every $a$ the matrix is circulant. Every time-invariant system on the finite-dimensional space of periodic discrete signals can be represented as a circular convolution.

The eigenvectors of the cyclic shift matrix are exponential signals, and the eigenvectors of circulants are the same exponential signals. Circulant matrices can be simultaneously diagonalizable (by the unitary Fourier matrix or discrete Fourier transform), and in general any commuting family of matrices is a simultaneously diagonalizable family [6]. Do similar conclusions hold for infinite-dimensional spaces?

The answer is in general negative. Characterizing timeinvariant linear bounded operators from $L_{p}(T)$ to $L_{q}(T)$ is not trivial, and the general case is still not completely understood. See [7] for a recent treatment and a characterization for $p=q=1$ and $p=q=2$. The following remark might be useful: if two linear systems $A$ and $B$ commute, they will have a common eigenvector if at least one of the two systems has an eigenvalue of finite multiplicity. To see why note that the set of eigenvectors of $A$ corresponding to the eigenvalue $\lambda$ is in the null space of $A-\lambda I$. Because $A-\lambda I$ commutes with $B$, its null space is invariant under $B$. If the dimension of this invariant space of $B$ is finite it must contain an eigenvector of $B$, and this will be a common eigenvector of $A$ and $B$, because it satisfies both $B x=\alpha x$ and $(A-\lambda I) x=0$.

We now consider the question raised in [1], and show that there exist interesting systems that are neither linear nor time-invariant and that have exponential eigenfunctions. It is helpful to consider first the following very simple case, upon which more complex classes of systems will be built. Let $F$ be a nonlinear and nonhomogeneous functional, and $H$ a LTI system. Consider the nonlinear, nonhomogeneous system defined by

$$
y=F[x] H x
$$

The term $F[x]$ can be considered as a signal-dependent gain, which affects the output of the LTI system $H$. Consequently, the system defined by (1) maps an exponential function into the same exponential, up to a complex factor.

For example, the output that corresponds to the exponential $x(n)=a^{n}$ is (using a suggestive but rather loose notation) $y(n)=F\left[a^{n}\right] H a^{n}$, the same exponential up to a complex factor (note that $F\left[a^{n}\right]$ is a complex number, the image under the functional $F$ of the signal $x(n)=a^{n}$, and $H a^{n}$ is proportional to $a^{n}$ because of the constraints imposed upon $H)$.
It should be clear now that $H$ need not be LTI. The exponential invariance subsists if $H$ is a system that has the exponential eigenfunction property but is not necessarily linear (in particular, $H$ can be HTI).

We are now ready to discuss the following proposition.

Proposition 1: Consider the system

$$
S[x]=\sum_{i \in J} F_{i}[x] H_{i} x
$$

where $J$ is a finite index set, the $F_{i}$ are functionals (not necessarily linear, nor time-invariant) and the $H_{i}$ are LTI systems. The response of the system $S$ to an exponential signal is the same exponential, up to a complex factor.

Proof: Assume that $x$ is exponential, say, $x(n)=a^{n}$. Since, by hypothesis, $f_{i}=H_{i} x$ is proportional to $x$ [say, $\left.f_{i}(n)=c_{i} a^{n}\right]$, the $n$th sample of the output of the system will be

$$
y(n)=\left(\sum_{i} F_{i}[x] c_{i}\right) a^{n}
$$

which shows that $y$ is the exponential $x$ up to the factor inside parentheses.

Again, the hypotheses concerning $J$ and the systems $H_{i}$ could be weakened, but for brevity we refrain from doing so. As a simple example, we might have imposed upon $H_{i}$ the weaker condition of homogeneity, and extending the results to continuous-time systems is straightforward.

Proposition 2: If all the functionals $F_{i}$ in (2) are timeinvariant, the system will be time invariant.

Proof: Since all the $F_{i}$ and $H_{i}$ are time-invariant, the response to $D x, D$ being an arbitrary delay, is

$$
S[D x]=\sum_{i} F_{i}[D x] H_{i} D x .
$$

But $H_{i} D=D H_{i}$ and $F_{i}[D x]=F_{i}[x]$, by definition, and it follows that

$$
S[D x]=\sum_{i} F_{i}[D x] D H_{i} x=D \sum_{i} F_{i}[x] H_{i} x=D S[x]
$$

which means that $S$ is time-invariant.

The following examples show that the class of systems expressed by (2) is not empty, and contains as special cases filter structures commonly met in signal processing.

Example 1: Let $F_{i}[x]=a_{i}$ (complex constants), and $H_{i}=$ $D_{i}$ (delay by $i$ units). The response $y$ of the system (2) to the input $x$ will then be given by

$$
y(n)=\sum_{i} a_{i} x(n-i) .
$$

Thus, the class of systems considered contains the finite impulse response (FIR) filters as special cases.

Example 2: Let $F_{i}[x]=a_{i}$ (complex constants), and $H_{i}=$ $H^{(i)}$ (composition of a fixed system $H, i$ times). The response $y$ of the system (2) to the input $x$ will then be given by

$$
y(n)=\sum_{i} a_{i} H^{(i)} x .
$$

The systems considered thus contain the generalized feedforward filters [8] as particular cases. 
Example 3: Take the sum in (2) to consist of only one term, or, equivalently, go back to (1). Let $H$ be an arbitrary LTI system and let

$$
F[x]=1+x(0) .
$$

The functional $F[x]$ is clearly time-varying, and so is the system $S[x]=F[x] H x$. The system is in addition nonlinear and nonhomogeneous, yet when the input is an exponential $x(n)=a^{n}$ one has $F[x]=1+a^{0}=2$ and the output becomes

$$
y=2 H x
$$

which is proportional to the exponential $x$ because $H$ is LTI.

Many other similar examples can be given. It is easy to obtain, for example, homogeneous systems of degree $n$, $S[\alpha x]=\alpha^{n} S[x]$, with exponential eigenfunctions.

\section{ACKNOWLEDGMENT}

The issues discussed in this letter came partly out of the author's teaching experience at the University of Aveiro, Por- tugal. The author's discussions with his colleagues, T. Oliveira e Silva, A. Pinho, and J. Vieira, are gratefully acknowledged.

\section{REFERENCES}

[1] P. P. Vaidyanathan, "Homogeneous time-invariant systems," IEEE Signal Processing Lett., vol. 6, pp. 76-77, Apr. 1999.

[2] L. V. Kantorovich and G. P. Akilov, Functional Analysis. New York: Pergamon, 1982.

[3] P. Hughett, "Linearity and sigma-linearity in discrete-time linear shiftinvariant systems," Signal Process., vol. 59, pp. 329-333, 1997.

[4] S. Boyd and L. O. Chua, "Fading memory and the problem of approximating nonlinear operators with Volterra series," IEEE Trans. Circuits Syst., vol. CAS-32, pp. 1150-1161, Nov. 1985.

[5] I. W. Sandberg, "A representation theorem for linear systems," IEEE Trans. Circuits Syst., vol. 45, pp. 578-580, May 1998.

[6] R. A. Horn and C. R. Johnson, Matrix Analysis. Cambridge, U.K.: Cambridge Univ. Press, 1990

[7] J. Ramanathan, Methods of Applied Fourier Analysis. Boston, MA: Birkäuser, 1998.

[8] T. Oliveira e Silva, "Stationary conditions for the $L^{2}$ error surface of the generalized orthonormal basis functions lattice filter," Signal Process., vol. 56, pp. 233-253, Feb. 1997. 